\title{
Equilibrio del dominio afectivo profesor estudiante en la enseñanza aprendizaje de la Matemática de la Educación Básica Superior
}

\author{
Balance of the affective domain student teacher in the \\ teaching learning of the Mathematics of the Superior Basic \\ Education
}

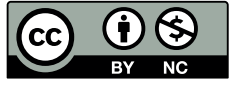

Msc. Tanya Navarrete Fernández ${ }^{1}$ tanyanavarrete2002@gmail.com

\section{RESUMEN}

Recibido: 1/07/2017, Aceptado: 1/09/2017

El estudio de la matemática siempre ha estado correlacionado a la idea de que es una disciplina con gran utilidad, pero también muy difícil de aprender en vista de la cantidad de conceptos que involucra. No solo es nuestro alumno el que tiene el reto de aprobar una materia; también lo somos los profesores quienes tenemos que luchar con las diferentes actitudes, creencias y emociones que ellos traen, y adaptar no solo nuestras metodologías sino también nuestras propias emociones, creencias y actitudes para forjar en el estudiante nuevas enseñanzas de acuerdo a su realidad actual y a las expectativas requeridas en el sistema educativo vigente. Son objetivos de este artículo revisar la profundidad e influencia en el aprendizaje marcados por los descriptores de los dominios afectivos de estudiantes, diseñar un instrumento para evaluar la influencia que los dominios afectivos producen tanto en el maestro como en el alumnado en el momento de la enseñanza-aprendizaje y la importancia del mantenimiento del adecuado equilibrio de los dominios afectivos presentes en el proceso. Se finaliza con los resultados y conclusiones obtenidas de las encuestas y el resultado de un cambio en la concepción de la matriz educativa basándose en nuevos paradigmas fundamentados en el diseño de pedagogías que incorporen la comunicación del conocimiento teniendo un sustento en valores humanos junto al entretenimiento al aplicar la tecnológica en la parte investigativa.

Palabras Clave: Dominio Afectivo, matemáticas, estudiantes, noveno curso, profesores

\begin{abstract}
The study of mathematics has always been correlated to the idea that it is a discipline with useful but also very difficult to learn in the light of the number of concepts involving. Not only is our student who has the challenge of passing a subject; also we are teachers who have to contend with different attitudes, beliefs and emotions that they bring, and adapt not only our methodologies but also our own emotions, beliefs and attitudes to build student new teachings according to their current reality and expectations required in the existing educational system. Goals of this article
\end{abstract}

\footnotetext{
${ }^{1}$ Estudiante de doctorado en Educación, Master en Evaluación y Diseño de Modelos Educativos, licenciada en Ciencias de la Educación en la especialización de Físico-Matemático de la Universidad Estatal de Guayaquil y Oceanógrafa graduada en la Escuela Superior Politécnica del Litoral (ESPOL), profesora de Bachillerato y Educación Básica Superior de la Unidad Educativa Bilingüe La Moderna. Ecuador
} 


\section{Jornada de Investigación Universidad Tecnológica Empresarial de Guayaquil}

are review the depth and influence on learning marked by descriptors of student's affective domains, design an instrument to evaluate the influence of affective domains produce both the teacher and students at the time of the teaching-learning and the importance of maintaining the proper balance of affective domains present in the process. Ends with the results and conclusions drawn from the polls and the result of a change in the conception of the educational matrix based on new paradigms based on the design of pedagogies that incorporate the communication of knowledge based on human values along with entertainment by applying the technology in the research part.

Keywords: Affective domain, mathematics, teachers, students, ninth course

\section{Introducción}

La enseñanza y el aprendizaje de las matemáticas ha sido objeto de múltiples estudios para dotar a esta ciencia de pedagogía y didáctica que desarrolle actitudes, destrezas que revelen interés de los educandos por aprenderla y que los maestros puedan llegar a alcanzar niveles de rendimiento académico aprobables en el sistema educativo. También es verdad que hemos estado conscientes de que todo lo afectivo procedente de la metacognición y las dimensiones afectivas de nuestros educandos han determinado como han sido estos rendimientos educativos, pero que esto siempre ha sido minimizado en la educación tradicional. En la realidad somos, como los griegos decían, cuerpo y psyche (alma, espíritu) todo en uno, y por lo tanto debemos al abordar temas educacionales, enfrentar las problemáticas como un todo. El maestro es un narrador, es una experiencia de vida con temporalidad y diferentes interpretaciones de acuerdo a la biografía de cada uno de sus alumnos. Y los alumnos son biografías en constante desarrollo, evolución y reconfiguración, en donde cada maestro deja su huella de conocimientos y vida, dándoles una reinterpretación propia, ayudándoles a ordenar, comprender y reprocesar información preconcebida para elaborar nuevos conocimientos y con ello, nuevos textos con direcciones y sentidos propios.

Gómez $(1997,2001)$ en base a los trabajos generados en el campo de las Didácticas de las Matemáticas por McLeod en 1988 ya tomaron en cuenta la dimensión afectiva en el aprendizaje de las matemáticas, ponen de manifiesto que las cuestiones afectivas juegan un papel esencial en la enseñanza aprendizaje de las matemáticas, y que algunas de ellas están fuertemente arraigadas en el sujeto, y no son fácilmente desplazadas por la instrucción.

Mayer, Caruso \& Salovey (1990) junto a Goleman (1995) hablan en cambio de una alfabetización emocional del educando para esta dimensión a tratarse en la Didáctica de la matemática. Se hace evidente que la relación entre el dominio afectivo y el aprendizaje no va en sentido único ya que la afectividad condiciona e interviene en el comportamiento y capacidad para aprender (Romero, Utrillas, \& Utrillas, 2014).

Gil, Blanco \& Guerrero $(2005,2006)$ trabajan este punto de vista de dominio afectivo o afecto y los descriptores básicos que para ellos rigen en cuanto a lo emocional, actitudinal y de creencias del educando como factores básicos en la comprensión y rendimiento en la materia. 


\section{Jornada de Investigación Universidad Tecnológica Empresarial de Guayaquil}

Molera (2011) plantea la importancia de que estos descriptores mencionados por Gil, Blanco \& Guerrero (2006) sean identificados y recogidos por medio de un instrumento diseñado para este propósito adecuado al nivel de su estudio.

Hidalgo, Maroto, Ortega \& Palacios (2013) trabajan lo afectivo en cuanto a los fracasos observados al observar una baja incidencia de los estudiantes por escoger carreras técnicas debido a los bajos niveles en las pruebas PISA sobre todo en matemáticas dados en España.

Ponen en relevancia lo que en los ochentas fue uno de los objetivos a alcanzarse en cuanto a la enseñanza de la matemática esto es, la metacognición para la resolución de problemas matemáticos. Sin embargo, posteriormente otros autores citados por Hidalgo, Maroto, Ortega \& Palacios (2013) dan a conocer que la dificultad más bien parece residir en que los estudiantes no logran discernir la forma de utilizar el conocimiento correcto ni cuando hacerlo, por lo que la metacognición de las estrategias y no las estrategias en si vienen a ser lo más importante para ayudar a mejorar el rendimiento de los estudiantes.

En nuestro país, Ecuador, Molina (2012) en su estudio sobre los "Factores de la Actitud y Ansiedad al aprendizaje de la matemática en estudiantes adolescentes en la ciudad de Milagro-Ecuador", recalca la relación de la estructura familiar, como aspecto afectivo que influye en el rendimiento académico causando ansiedad matemática en el alumno, ya que viene por la familia la presión de poder rendir adecuadamente en una materia que exige precisión; denotando una falta de confianza en sí mismo. Adicionalmente factores de tipo familiar como el divorcio de sus padres y dificultades económicas también suponen una presión que genera la ansiedad traducida como dificultad matemática y que se ven reflejadas en las estadísticas de los indicadores educativos del ministerio de Ecuador 2011-2012 en donde los porcentajes de pérdidas de año son un 3,3\%.

¿Qué se entiende por Dominio Afectivo?

El Dominio Afectivo de acuerdo a Krathwohl, Bloom \& Masia (1973) creadores de la Taxonomía de los Objetivos de la Educación, son todas las actitudes, creencias, apreciaciones, gustos, preferencias, emociones, sentimientos y valores con las que un individuo se identifica y que se reflejan en el momento de actuar durante el proceso del aprendizaje.

McLeod \& McLeod (1982) se refiere a este término como un extenso rango de sentimientos y humores (estados de ánimo) que son generalmente considerados como algo diferente de la pura cognición, e incluye como componentes específicos de este dominio, las actitudes, creencias y emociones.

¿Cuáles y como se definen estos descriptores en el dominio afectivo?

Los descriptores básicos son lo emocional, sus creencias y lo actitudinal. Todos ellos se vinculan íntimamente.

Así, de acuerdo con Gómez (2002), las creencias matemáticas forman parte del conocimiento subjetivo implícito del individuo se basan en la experiencia en las matemáticas, su enseñanza, aprendizaje tanto del alumno como del profesor y son inconscientes. 


\section{Jornada de Investigación Universidad Tecnológica Empresarial de Guayaquil}

Las actitudes "son experiencias subjetivas (cognitivo-afectivas) que implican juicios evaluativos, que se expresan en forma verbal o no verbal, que son relativamente estables y que se aprenden en el contexto social" (Díaz \& Hernández, 2010, p. 45). Por lo tanto, las actitudes se las puede entender como el comportamiento positivo o negativo producto de una experiencia que se manifiesta como acciones o sentimientos de aceptación o rechazo. Las emociones que se pueden traducir como sentimientos son respuestas a sucesos o eventos internos o externos que denotan frustración o realización ante lo conseguido en el campo del conocimiento matemático.

Según (Gil et al., 2005), "Las emociones son respuestas organizadas más allá de la frontera de los sistemas psicológicos, incluyendo lo fisiológico, cognitivo, motivacional y el sistema experiencial. Surgen en respuesta a un suceso, interno o externo, que tiene una carga de significado positiva o negativa para el individuo" ( $p$. 23).

\section{Objetivos}

Conocer los descriptores y valorar la profundidad de los mismos por medio de encuestas orientadas hacia el dominio afectivo imperante en las clases de los novenos años de educación básica en un colegio particular de clase media alta.

Diseñar un instrumento de medición en base a la problemática planteada para determinar el tipo de dominios imperantes en el aula y el tipo de equilibrio de dominios que se encuentran.

Consecuencias que se observan en la evolución de los grupos al existir una mayor interacción y modificación de los descriptores afectivos en el ambiente áulico.

\section{Preguntas de la investigación}

¿Cuáles son los descriptores básicos predominantes que se dan en el dominio afectivo en la materia de matemáticas durante las clases de los novenos años de educación básica? ¿Qué resultados nos arroja el medir el grado de influencia del maestro en el curso? ¿Qué resultados nos arroja el medir la influencia de los descriptores emocionales, actitudinales y de creencias en los estudiantes? ¿Cuáles son las consecuencias que se observan producto de la modificación de alguno de los descriptores observados en las clases de matemáticas?

Las clases de matemáticas en los novenos año de educación básica presentan inconvenientes tanto a nivel áulico por el microclima negativo que se da en el interior especialmente de un de los paralelos, así como en la dispersión que se produce en la atención del estudiante; por lo que la autora del presente documento se ve en la necesidad de investigar más a fondo la causa o causas circunstanciales que han producido este ambiente y desempeño áulico deficiente a nivel de la clases de matemáticas. Se observa que en otras materias como lenguaje, estudios sociales y ciencias naturales existe también un nivel de comportamiento bastante bajo, lo cual es relevante e influyente no solo a nivel del rendimiento escolar sino también a nivel social.

Las notas de los alumnos en promedio de noveno son bajas en las cuatro materias del eje curricular ya que se encuentran entre 7,58 en Lenguaje y Literatura y 5,41 en Matemáticas esto para el primer quimestre en uno de los paralelos. Para el mismo periodo en el otro paralelo para las mismas materias los puntajes se encuentran entre 


\section{Jornada de Investigación Universidad Tecnológica Empresarial de Guayaquil}

un máximo de 8,12 en Lengua y 6,35 como un mínimo en Matemáticas.

La investigación es viable en vista que es posible llevar a cabo el estudio y se dispone de los recursos humanos y técnicos previstos para su ejecución. Con este estudio se espera poder tener un conocimiento de la importancia de los dominios afectivos para con los sujetos del estudio y encontrar la forma de enfrentarlo y minimizar sus efectos en el interior de las aulas para transformar en medida de lo posible los efectos negativos que se reflejan en el rendimiento escolar.

\section{Desarrollo}

En el actual gobierno del presidente Econ. Rafael Correa el Ecuador ha invertido dos veces más que anteriores gobiernos en el rubro de la educación propendiendo a subir los porcentajes de rendimiento escolar a nivel de las cuatro materias de evaluación como son Matemática, Lenguaje, Estudios Sociales y Ciencias Naturales a nivel de América Latina por encima de países como Perú, Colombia, Argentina que experimentan los más bajos rendimientos. Hay que reconocer que hasta hace pocos años atrás nuestro país obtenía valores por debajo de la media aritmética de los países Latinoamericanos. Hoy por hoy la materia de Matemáticas ostenta un puntaje de 520 lo que hace que se avale lo instaurado por el gobierno en materia de Educación.

Más, sin embargo, las políticas de estado en lo educativo aún no complementan concretamente lo subjetivo en la educación como son los dominios afectivos en materias como matemáticas sobre todo y en ciencias en general, donde se deberían tener en cuenta dependiendo de las circunstancias específicas y definidas a cada situación regional y local en el país.

Esta investigación se contextualiza en dos paralelos de noveno año de educación básica de un colegio particular de la ciudad de Guayaquil, en el año lectivo 20162017. Los individuos a ser evaluados son estudiantes ecuatorianos adolescentes de entre 12 y 14 años de clase media alta a los cuales se los ha investigado cualitativamente. Se observa su aspecto actitudinal (notas de comportamiento) al igual que su aspecto cognitivo registrado a través de las notas que arrojan en la materia de matemáticas durante el primer quimestre del año lectivo en curso.

Se comprueba que los promedios en rendimiento académico para ambos paralelos son bajos en la materia de matemática siendo en un primer paralelo de 6,35 y en el otro de 5,41. Las notas por comportamiento en cada uno de los parciales arrojan promedios quimestrales de 7,63 y 7,29 .

Se hacen observaciones áulicas que pasan desapercibidas por la cotidianidad con que la autora las registra y recepta las particularidades de estos grupos.

La observación y exploración áulica de los educandos durante 6 meses nos permiten identificar lo siguiente:

- El horario en que se dictan materias de ciencias y exclusivamente de matemáticas a estos cursos de noveno es pasadas las primeras horas de la mañana, esto es después de las nueve de la mañana, a excepción de una hora del noveno A registrada a primera hora de los martes.

- La temperatura promedio en Guayaquil es alta y con humedad por lo que los ambientes áulicos se tornan calurosos y la deshidratación de los educandos se 


\section{Jornada de Investigación Universidad Tecnológica Empresarial de Guayaquil}

produce con mucha frecuencia.

- Salones con ciertos alumnos sin un autocontrol ante la presencia del maestro y peor sin la presencia del mismo, ya que se deriva la indisciplina hacia todos los educandos.

- La indisciplina en el salón de clases encierra factores como desaseo del aula con elementos como materiales de estudio llámense hojas, cuadernos o libros. También se da el desaseo por envolturas de alimentos o alimentos en si caídos y mochilas en los pasillos.

- En la parte actitudinal de los educandos cuando están solos temporalmente o durante los cambios de hora se desmerece mucho pues se paran, salen del salón, se agreden física y verbalmente, gritan, ríen en forma estrepitosa, conversan, pelean, dañan materiales del aula y de los compañeros.

- Cuando se encuentran en clase estos grupos denotan una clara falta de respeto para con sus profesores, pues a pesar de ser advertidos de la forma correcta en como intervenir simplemente responden al unísono de manera que es otra forma de bulla y desorden maximizada por la existencia de educandos que se paran con cualquier pretexto sin solicitarlo oportunamente a su maestro de manera que interrumpen la clase creando la distracción al resto de compañeros.

- Por los expedientes de conducta revisados se pueden detectar otros casos particulares de indisciplina que no son un común denominador sino más bien casos aislados, y que deberían ser parte de otro tipo de planteamiento.

\section{Método}

El método a emplear para comprender y profundizar en el estudio con que intervienen la actitud y las creencias de los educandos es explorando la perspectiva subjetiva de los participantes en su ambiente áulico cotidiano es el cualitativo. El mismo que será inductivo partiendo de los objetivos uno y tres. Con este fin se usaron las observaciones directas que fueron concertadas en aula como ya se mencionó.

Con el segundo objetivo planteado la investigación se plantea un instrumento como es la encuesta de tipo deductiva ya que se la estableció partiendo de lo observado para medir la influencia de dichos descriptores, de manera que pruebe las hipótesis planteadas, así como evaluar los efectos de unas variables sobre otras de manera correlacionada.

Se desarrolló en base a los resultados un análisis estadístico descriptivo de la muestra y se aplicó un análisis crítico socio-educativo de la muestra que pretende desarrollar una revisión de los parámetros y conceptos con los que los maestros debemos incursionar en las biografías de nuestros pupilos ayudando a transformar esta problemática por medio de estrategias pedagógicas de contenido afecto-constructivo.

Se presentan los resultados de las encuestas a los estudiantes y entrevistas con profesionales docentes del área de matemáticas.

Las conclusiones están orientadas a describir el tipo de equilibrio de dominios afectivos en el aula y como cambiar el paradigma educativo con grupos heterogéneos en sus creencias, emociones y actitudes.

\section{Metodología}

Se tomó una muestra de la Educación Básica Superior inferior a 100 estudiantes, es decir 56 estudiantes de dos cursos de noveno año en un colegio particular de la ciudad, entre hombres y mujeres, con edades entre los 12 y 14 años. Ninguno con 


\section{Jornada de Investigación Universidad Tecnológica Empresarial de Guayaquil}

informe de algún tipo de discapacidad física o mental presente reconocida.

Los tipos de muestras utilizadas en esta investigación son las no probabilísticas, heterogéneas ya que se presentan casos donde los estudiantes muestran actitudes desde muy tranquilos hasta exageradamente activos.

Instrumento: el cuestionario utilizado se basa en preguntas registradas hacia las relaciones interpersonales en la clase con los compañeros (ambiente áulico), la actitud del profesor percibida por el alumno y el grado de utilidad y de agrado que la matemática le podrá significar personalmente en su vida futura.

Se solicitó la ayuda de un maestro de otro paralelo en el área de matemáticas para que receptara la evaluación y no hubiera ninguna injerencia por parte de la presencia del profesor de la materia.

El tiempo de la encuesta es de una hora clase.

Factor encuestado: actitud del profesor en clase. -

Se pretende entender las relaciones con el profesor de la materia y como lo observan durante sus clases. Entre las actitudes encuestadas están:

a) ¿Me anima a estudiar y comprender matemáticas?

b) ¿Cómo me siento motivado(a) a aprender matemáticas?

c) ¿Siento que el profesor le gusta enseñarnos?

d) ¿Me siento en confianza para preguntarle a mi profesor(a) sino entiendo algo?

e) ¿Con sus actitudes me hace pensar que puedo ser mejor en matemáticas?

f) ¿Siento que le intereso como alumno en sus clases?

g) ¿Me gusta cómo enseña?

h) ¿Me dice en que me equivoco y cómo hacer para no volver a equivocarme? ¿Me hace ver mis errores?

i) ¿Se interesa en ayudarme con mis dificultades? ¿Se preocupa por mis notas?

j) ¿Se preocupa porque participe en clases? ¿Se preocupa de que entienda su clase?

Factor encuestado: utilidad y agrado por las matemáticas. -

Se pretende conocer si están conscientes de la utilidad de las matemáticas y si tienen una predisposición natural por las mismas.

a) ¿Creo que las matemáticas serán importantes para mi futuro profesional?

b) ¿Actualmente las matemáticas son importantes y útiles para mi vida diaria?

c) ¿Entiendo los deberes y proyectos que se me envía en clase?

d) ¿En años anteriores me gustaban las matemáticas?

e) ¿Considero que se me hacen fáciles las matemáticas? ¿Tengo aptitudes para las matemáticas?

f) ¿Creo que podría enseñar a otras matemáticas?

g) ¿Me veo en el futuro con una carrera relacionada a las matemáticas? ¿Cuál carrera podría ser?

Factor encuestado: actitudes de los educandos con los docentes en el aula, punto de vista del maestro. -

Se pretende auscultar la relación educando-docente desde los dominios afectivos del 


\section{Jornada de Investigación Universidad Tecnológica Empresarial de Guayaquil}

docente por medio de la siguiente entrevista:

a) ¿Le gusta ser maestro?

b) ¿Se divierte con sus estudiantes dando clases?

c) ¿Cree usted que es un buen profesor?

d) ¿Cree usted que las matemáticas son necesarias para la formación integral de sus educandos?

e) ¿Cómo se califica como profesor en una escala del 1 al 10 siendo uno malo y 10 sobresaliente?

f) ¿Cuál cree usted que es su mayor virtud como docente?

g) ¿Cree que sus alumnos le entienden?

h) ¿Porque cree que fue educador y no medico por ejemplo?

i) ¿Se interesa porque sus alumnos le entiendan?

j) ¿Interactúa con sus estudiantes fuera de clases?

k) ¿Cree usted que sus alumnos se interesan en sus clases?

1) ¿Considera que en sus clases hay bullying entre sus alumnos?

m) ¿Cómo reacciona ante un curso bullicioso, sucio y con alumnos sin autocontrol?

n) ¿Con cuanta frecuencia se enoja? En la escala de 1 a 10, siendo 1 nunca y 10 siempre

o) ¿Cuáles serían los motivos por los que se enojara?

\section{Resultados}

Las encuestas realizadas tuvieron como finalidad medir la forma en que los estudiantes tienen la percepción de su maestro. La empatía del estudiante hacia su profesor es un factor decisivo para que el alumno se embarque en la aventura de aprender y querer hacerlo de manera decisiva.

De los resultados arrojados por el cuestionario aplicado a los estudiantes de noveno sobre el factor actitud del profesor hacia los educandos, observamos que existe una buena predisposición desde y hacia el maestro de matemáticas y que llega con una percepción positiva de parte de sus alumnos, en su mayoría con un porcentaje por encima del $70 \%$ como podemos observar en el gráfico 1 .

El puntaje más bajo en la percepción de los educandos es la manera en la que enseña. Investigando los motivos por los cuales puede darse esta respuesta y en un análisis de la metodología expuesta en los planes de destrezas desarrollados para ese año lectivo, se observa que las destrezas no se logran en un $40 \%$ ya que existe un porcentaje detectado de estudiantes que para ese nivel no logran un dominio completo en las cuatro operaciones básicas de la aritmética. Además, los procesos sobre las operaciones básicas en números enteros presentan muchas falencias tanto en su ejecución (proceso) como en los resultados (cognitiva y actitudinal) durante las clases. Estos inconvenientes no superados en su formación en años anteriores se reflejan claramente al momento de involucrar conceptos más elaborados como son los números reales y podrían ser el motivo al momento de la encuesta. 
Revista Ciencia \& Tecnología No. 16, 31 de octubre de 2017 ISSN impreso: 1390 - 6321

\section{Jornada de Investigación Universidad Tecnológica Empresarial de Guayaquil}

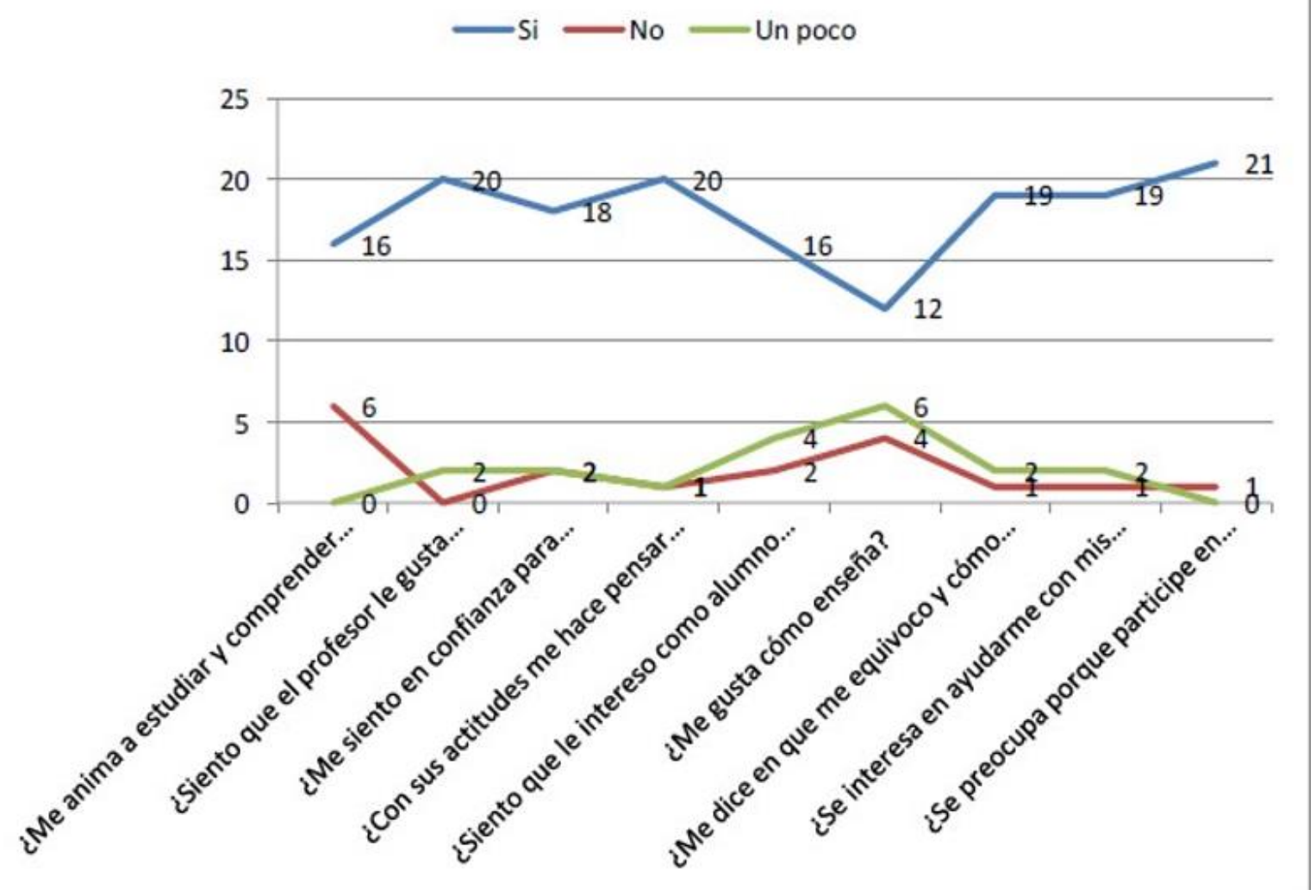

\section{Gráfico 1. Actitud del profesor: Medición de la percepción del alumno}

Fuente: sección EBS en Colegio particular

Elaborado: por Tanya C. Navarrete Fernández

En el segundo cuestionario aplicado al estudiantado sobre la utilidad e importancia que estos le dan a las matemáticas observamos que si bien es cierto la consideran importante, no le ven la utilidad de lo que han visto y por ende se muestran inseguros declarando que no hacen deberes o no los entienden pero que antes de este año lectivo si les gustaba las matemáticas o tenían predisposición para las mismas e incluso podrían hacer una futura carrera que tenga relación con las matemáticas, pero que sin embargo no se sienten capaces de enseñarlas (gráfico 2 ). 
Revista Ciencia \& Tecnología No. 16, 31 de octubre de 2017 ISSN impreso: 1390 - 6321

\section{Jornada de Investigación Universidad Tecnológica Empresarial de Guayaquil}

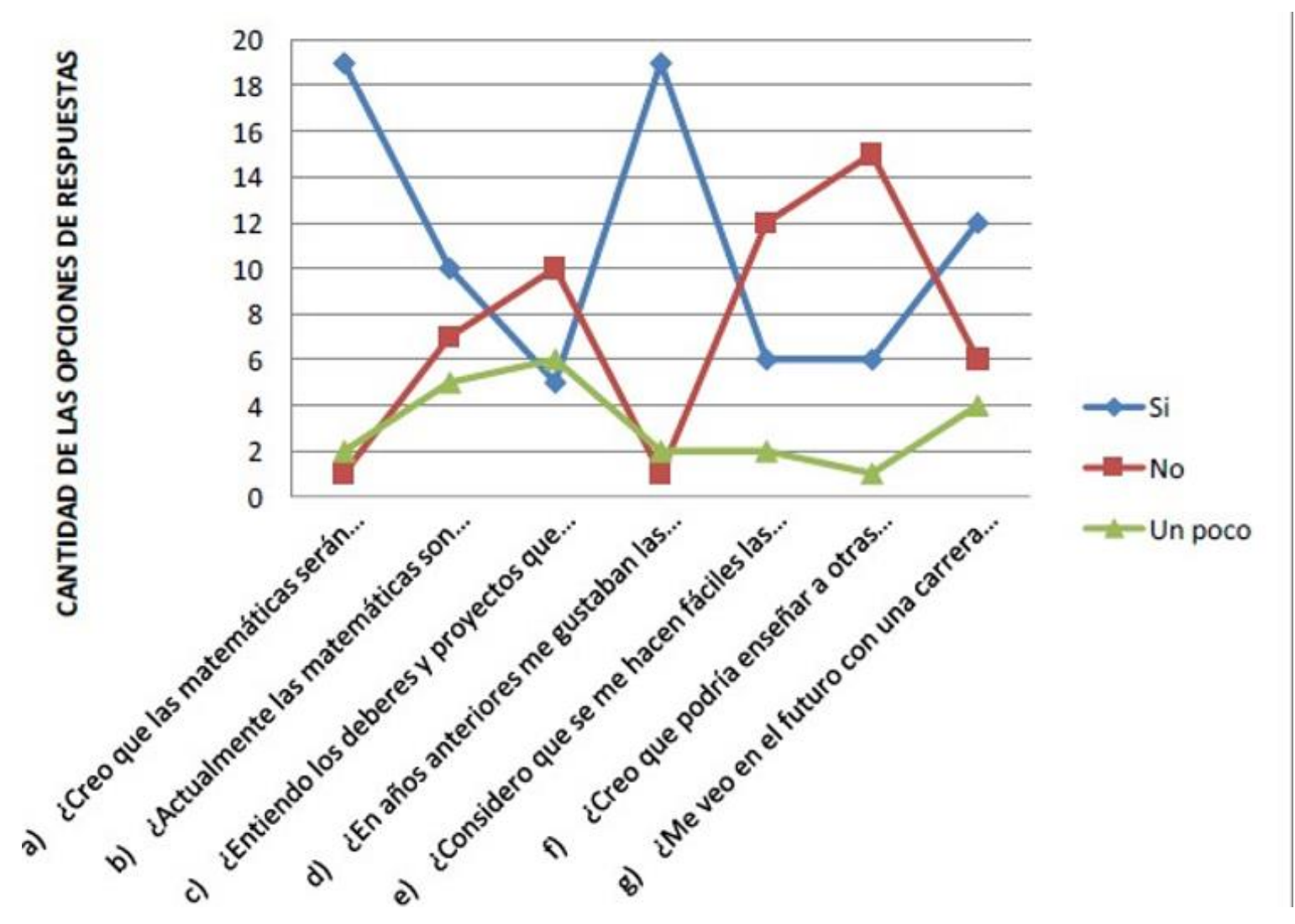

Gráfico 2. Utilidad y agrado por las matemáticas

Fuente: Sección EBS Colegio Particular

Elaborado por Tanya C. Navarrete Fernández

Finalmente, en la entrevista realizada a diferentes docentes del área de matemáticas se pudo concluir lo siguiente: Los maestros se encuentran involucrados con sus educandos ya que les gusta ser maestros, se divierten impartiendo clases, tratando de dar lo mejor de ellos en sus clases e implementando metodologías que les permitan llegar mejor a sus educandos con sus conocimientos, siendo la perseverancia y la paciencia las virtudes que destacan en su labor docente. Sin embargo, la parte actitudinal influencia mucho en el desarrollo de las clases, cuando se encuentran con aulas descuidadas por efecto de la basura, el desorden de bancas o los alumnos fuera de su lugar. La falta de autocontrol y valores humanos como el respeto es bastante marcada también en los cursos de noveno de básica.

\section{Conclusiones}

Ante este cambio de paradigmas en que se encuentra inmerso el actual sistema educativo ecuatoriano que nos presenta un estudiante con unas características detectadas en este estudio nunca antes vistas como el irrespeto entre estudiantes y hacia el maestro, la ignorancia y el aburrimiento, un franco deterioro de las actitudes positivas; es necesario llevar a la escuela lo que el Dr. Jorge Noro en su cuarta jornada del doctorado de Octubre, 2016 expuso como Propuestas y Sugerencias 


\section{Jornada de Investigación Universidad Tecnológica Empresarial de Guayaquil}

para una post escuela en un mundo educativo moderno, que lo implementaremos como fases.

Los dominios afectivos en el profesor de la materia deben ser lo suficientemente firmes, sólidos, definidos para enfrentar grupos con situaciones emocionales muy diversas y transformar los aspectos negativos del grupo en aspectos positivos rescatando las aptitudes innatas en los estudiantes, sus creencias y sentimientos que los mueven. Por ser grupos muy heterogéneos es necesario buscar la narrativa que los una y el sentimiento que los embargue para poder llegar a tener un mayor grado de captación de voluntades en el querer aprender.

Fase 1. Diseño y planeación estratégica en la enseñanza de las matemáticas en contenidos y metodología

Basados en los contenidos dados por el ministerio de Educación se propondrá que cada unidad sea en base a un proyecto educativo de análisis donde la metodología empleada sea de tipo deductivo, es decir exponiendo el resultado logrado se descompondrá el proceso hasta llegar a sus partes haciendo un análisis del porqué del mismo.

Recursos, lenguaje y escenarios

Los recursos serán dados en función de los resultados a obtener siempre haciendo la observación de emplear los términos apropiados para cada elemento o término matemático empleado junto a la parte simbólica o rasgo matemático identificativo del término. Aquí el estudiante podrá desarrollar su creatividad en cuanto al medio en el cual se desarrolla su proyecto dándole realce a la parte matemática involucrada en su escenario.

Ordenamiento, sistematización de saberes, información y comunicación de saberes

Todos los conocimientos extraídos de la experiencia deberán ser plasmados e incorporados bajo un esquema propio reconstruido por el alumno siempre guiados por una guía dada por el maestro donde podrá comunicar y brindar la información al resto del curso. Es importante también que con esto se haga una evaluación propia y del grupo al cual va dirigido esta información, lo cual dará paso a un instrumento.

\section{Instrumento de acreditación}

Este instrumento de acreditación deberá no solo contener la evaluación anteriormente mencionada sino también la creación de propuestas futuras y la manera en que dicho conocimiento pueda ser aplicado en otras áreas o ramas del saber; ya que las matemáticas tienen diferentes direccionamientos en cuanto a aplicación.

Fase 2. Desarrollo de la personalidad del educando por medio de la transmisión del conocimiento y de la cultura de valores

Todos los seres humanos somos capaces de transmitir conocimiento por lo tanto solo es necesario que ayudemos todos los maestros de un determinado nivel a nuestro educando a descubrir cómo hacerlo, es tratar de leer el texto por medio de su biografía y desarrollar los aspectos potenciales que guarda en su interior, racionalizando y construyendo aspectos no tan relevantes en su personalidad. Es aquí donde también debe actuar el departamento de Orientación de los colegios (DECE) para sustentar y recomendar los aspectos a tomar en cuenta. 


\section{Jornada de Investigación Universidad Tecnológica Empresarial de Guayaquil}

La idea es que el educando en sí mismo se convenza y convenza a los demás que es posible aprender matemáticas sin que sea una parte tabú o compleja de aplicar. Y que hay muchos caminos para aprender todos con diferentes formas, pero todas validadas en la perspectiva personal de cada educando. Para esto es necesario que intervengan aquí los dominios afectivos tanto del educando como del educador, el uno para crear y el otro para guiar esta fase.

Fase 3. El entretenimiento y la supervisión en el desarrollo del conocimiento El alumno debe manejar este proceso de aprendizaje de manera semiautónoma basado en la guía que dio en clase el maestro. El maestro debe ser hábil para incorporarse en el aprendizaje del alumno, pero solo como una guía poniendo en manifiesto sus propios dominios afectivos y pedagógicos para lograr encaminarlo.

Es importante que siempre se establezcan los objetivos a lograr o el fin al cual deben llegar sus estudiantes por lo que deberá ser claro, lógico en la metodología a aplicar y el cómo examinar y concluir en los resultados obtenidos. Es decir deberá llevar al estudiante a la crítica y autocrítica de los resultados obtenidos por él y si se lograron cumplir los objetivos planteados por el maestro.

El estudiante deberá disfrutar procesando, aprendiendo, creando y siendo capaz de incorporar otros saberes como los tecnológicos en el logro de llegar a hacer suyos esos saberes y poder ser gestor de nuevas formas de definir y lograr conocimientos.

Fase 4. La investigación y la gestión docente

Al vincular la investigación en el proceso de aprendizaje, el maestro deberá gestionar la dirección de nuevos conocimientos que se articulen al sistema de lo visto en clases de manera haya nuevas interrogantes en los estudiantes y que los oriente hacia la realidad en su aplicación dinámica y decidida.

\section{Referencias bibliográficas}

Caballero, A., Blanco, L. J. \& Guerrero, E. (2007), Las actitudes y emociones ante las Matemáticas de los estudiantes para Maestros de la Facultad de Educación de la Universidad de Extremadura. Simposio de Investigación y Educación Matemática, Universidad de La Laguna.

Díaz, F. \& Hernández, G. (2010). Estrategias docentes para un aprendizaje significativo. México. McGraw-Hill.

Gil, N., Blanco, L. \& Guerrero, E. (junio, 2005). El dominio afectivo en el aprendizaje de las matemáticas. Una revisión de sus descriptores básicos. Unión. Revista Iberoamericana de Educación Matemática, 2, 15-32. Recuperado de http://www.fisem.org/www/union/ revistas/2005/2/Union_002_004.pdf

Gil, N., Guerrero, E. \& Blanco, L. (enero-abril, 2006). El dominio afectivo en el aprendizaje de las matemáticas. Revista Electrónica de Investigación Psicoeducativa, 4(1), 47-72. Recuperado de http://www.investigacionpsicopedagogica.org/ revista/articulos/8/espannol/Art_8_96.pdf

Goleman, D. (1995). Inteligencia Emocional. Barcelona: Kairós. 


\section{Jornada de Investigación Universidad Tecnológica Empresarial de Guayaquil}

Gómez, I. (1997) Procesos de aprendizaje en matemáticas con poblaciones de fracaso escolar en contextos de exclusión social. Las influencias afectivas en el conocimiento de las matemáticas. Tesis Doctoral, Universidad Complutense de Madrid.

Gómez, I. (2003) La Tarea Intelectual en Matemáticas Afecto, Meta- afecto y los Sistemas de Creencias. Boletín de la Asociación Matemática Venezolana, Vol X, No. 2, 225.

Hidalgo, S., Maroto, A., Ortega, T. \& Palacios, A. (2013). Atribuciones de Afectividad hacia las Matemáticas. Unión, Revista Iberoamericana de Educación Matemática, 35: 93-113.

Disponible:

http://www.fisem.org/www/union/revistas/2013/35/archivo10.pdf

Krathwohl, D. R, Bloom, B. S, \& Masia, B. B. (1973). Taxonomía de los objetivos educativos, clasificación de los objetivos educativos. Manual II: Dominio afectivo. Nueva York: David McKay Co., Inc.

Martínez, O. (2007). Discusión Pedagógica. Actitudes hacia las matemáticas. Recuperado de https://biblat.unam.mx/es/revista/sapiens/articulo/discusionpedagogica-actitudes-hacia-la-matematica

Mayer, J. D., Caruso, D. \& Salovey, P. (1999). Emotional intelligence meets traditional standards for an intelligence. Intelligence, 27, 267-298.

McLeod, D. \& McLeod, S. (2002). Synthesis-Beliefs and Mathematics Education: Implications For Learning, Teaching, and Research [Síntesis-creencias y educación matemática: Implicaciones para el aprendizaje, enseñanza e investigación]. En G. Leder, E. Pehkonen, \& G. Törner (Eds.),

Molera, J. (2011). Importancia de los factores afectivos en las matemáticas de educación primaria. Elaboración de un instrumento de evaluación. Recuperado de http://infad.eu/RevistaINFAD/2011/n1/volumen3/INFAD_010323_345354.pdf

Molina, E. (2012). Factores de la Actitud y Ansiedad al aprendizaje de la matemática en estudiantes adolescentes en la ciudad de Milagro-Ecuador. Unión, Revista Iberoamericana de Educación Matemática, 29: 109-120. Disponible: http://www.fisem.org/www/union/revistas/2012/29/archivo10.pdf

Noro, J. (2016) Apuntes para clases del Doctorado en Educación: Propuestas y Sugerencias para una Post Escuela, Nuevo mundo educativo.

Romero, L., Utrillas, A., \& Utrillas, V., (2014). Las actitudes positivas y negativas de los estudiantes en el aprendizaje de las matemáticas, su impacto en la reprobación y la eficiencia terminal. Disponible en: http://www.redalyc.org/articulo.oa?id=46132134020 